\title{
A contemplation on the secondary origin of green algal and plant plastids
}

\author{
Eunsoo Kim ${ }^{1 *}$, Shinichiro Maruyama² \\ 1 Sackler Institute for Comparative Genomics and Division of Invertebrate Zoology, American Museum of Natural History, Central Park West at 79 Street, New York, NY 10024, USA \\ ${ }^{2}$ Division of Environmental Photobiology, National Institute for Basic Biology, Nishigonaka 38, Myodaiji, Okazaki 444-8585, Japan
}

\begin{abstract}
A single origin of plastids and the monophyly of three "primary" plastid-containing groups - the Chloroplastida (or Viridiplantae; green algae+land plants), Rhodophyta, and Glaucophyta - are widely accepted, mainstream hypotheses that form the basis for many comparative evolutionary studies. This "Archaeplastida" hypothesis, however, thus far has not been unambiguously confirmed by phylogenetic studies based on nucleocytoplasmic markers. In view of this as well as other lines of evidence, we suggest the testing of an alternate hypothesis that plastids of the Chloroplastida are of secondary origin. The new hypothesis is in agreement with, or perhaps better explains, existing data, including both the plastidal and nucleocytoplasmic characteristics of the Chloroplastida in comparison to those of other groups.
\end{abstract}

Keywords: Archaeplastida; Chloroplastida; glaucophytes; green algae; plastids; primary plastids; red algae; secondary plastids; Viridiplantae

\section{Introduction}

The origin and spread of eukaryotic photosynthesis via cell-to-cell merging was a major driving force in the diversification of eukaryotes [1]. About ten eukaryotic lineages originated, or had their diversification facilitated, as photosynthesis was acquired through the process of endosymbiosis: these include chlorarachniophytes, cryptophytes, chromerids+apicomplexans, dinoflagellates, euglenophytes, glaucophytes, green algae + land plants (together known as Chloroplastida or Viridiplantae), haptophytes, red algae, and stramenopiles [2] [note that the rhizarian Paulinella chromatophora is a photosynthetic eukaryote, but its photosynthetic organelle (i.e., chromatophore) has an independent origin from the canonical plastids of other eukaryotic algae]. Plastids of these algae were derived from cyanobacteria and are closely related to each other, to the exclusion of the known diversity of cyanobacteria, suggesting their single cyanobacterial ancestry [3,4]. Depending on the number of endosymbiotic transfers that have been made since they were free-living cyanobacteria, plastids are classified as primary, secondary, or tertiary [2]. Conceptually, a primary plastid refers to one that was acquired directly as a cyanobacterium by a host eukaryote; the origin of secondary or tertiary plastids

\footnotetext{
*Corresponding author. Email: ekim1@amnh.org
}

Handling Editor: Andrzej Bodył involves respectively two or three endosymbiotic transfers of these organelles, with the last one or two endosymbioses occurring between two eukaryotic organisms [1]. In general, primary plastids are identified by the presence of two layers of plastid envelope membrane; by contrast, secondary and tertiary plastids are usually bound by three or more layers of membrane, although exceptions exist [2,5]. The current dominant understanding is that three eukaryotic groups green algae+land plants, red algae, and glaucophytes (all three together classified as Archaeplastida) - originated from a single ancestral endosymbiotic event that involved a cyanobacterial endosymbiont and, as a result, harbor plastids of primary origin [6]. Subsequent to this event, it is believed that eukaryotic photosynthesis spread into other lineages of eukaryotes via secondary and tertiary endosymbioses, which occurred on multiple occasions [1]. Three independent acquisitions of green algal-derived plastids are known to date [7]; the exact number and nature of endosymbiotic events that generated red algal-derived plastids, however, remains less certain $[1,8,9]$.

Over the past several years, a number of studies have utilized a wealth of molecular sequence data for inferring relationships among major eukaryotic groups $[10,11]$. Interestingly (and frustratingly for some authors), the archaeplastidan monophyly has rarely been recovered in analyses based on nucleus-encoded proteins, unless taxon sampling is compromised. Given this observation as well as other lines of evidence, we suggest the testing of an alternative idea, which 
is similar to the hypothesis pertaining to the evolutionary origin of primary plastids proposed by Stiller [12,13], that the Archaeplastida hypothesis may be fundamentally flawed because "primary plastids" as we understand it are of mixed, serial endosymbiotic origins. If this proposed hypothesis is indeed correct, we suggest that, of the three archaeplastidan groups, plastids of the Chloroplastida (or Viridiplantae) are most likely to be of secondary origin. This is based on several lines of observation as elaborated below.

\section{Multi-gene based phylogenetic approaches thus far have not been able to unambiguously support the Archaeplastida hypothesis}

Analyses of plastid genomes and other plastid-associated features significantly support the monophyly of plastids to the exclusion of cyanobacteria, thereby suggesting that the plastids originated from a single cyanobacterium-like ancestor $[3,4,14-17]$. This, together with the assumption of a primary origin for the plastids of green algae (plus their land plant descendants), glaucophytes, and red algae, form the basis of the Archaeplastida hypothesis. This posits that the three archaeplastidan groups form a clade and originated from a single plastid-generating endosymbiosis [5]. Corroborating this, some of the earlier phylogenetic studies that utilized nucleus-encoded proteins seemed, at least partially, to support the Archaeplastida hypothesis with moderate to strong bootstrap support values (e.g. [18-21]). However, as more taxa or protein data (or both) were included, archaeplastidan monophyly dropped in support, or was not recovered at all (e.g. [10,11,22-31]). One recent study representative of this work reported the recovery of archaeplastidan monophyly based on analyses of fourteen mitochondrion-encoded proteins [32]. However, bootstrap support for this topology was weak, and, more importantly, archaeplastidan monophyly was not recovered when certain taxa, including the excavates Reclinomonas and Malawimonas - which had been shown to branch with archaeplastidan groups in earlier studies as well [33-35] - were included [32]. It has been suggested that the Archaeplastida hypothesis has been firmly supported by analyses comparing percentages of protein trees that significantly support the monophyly of each of its component taxa with a range of other major eukaryotic groups $[17,36]$. However, because these analyses did not take out-group or branch-order information into account, it is not clear how the resulting percentage values actually translate to support for hypotheses of archaeplastidan phylogeny. In fact, these results are also consistent with our proposed "serial" hypothesis because endosymbiotic gene transfers (EGTs) among the archaeplastidan groups could elevate the percentage values when archaeplastidan groups are compared to one another. In summary, phylogenetic inferences based on multiple nucleocytoplasmic markers do not significantly support, nor do they refute, the Archaeplastida hypothesis.

Nevertheless, the Archaeplastida hypothesis has been assumed to be correct by many (e.g., [6,17,37-40]). In this context, an interesting problem has arisen in the course of attempts to resolve internal relationships among the three groups comprising Archaeplastida [41]. That is, phylogenies based on plastid genomes were shown to be in conflict with trees inferred from nucleocytoplasmic data [41]. Such an observed discrepancy could be explained at least partially by one or two of the archeaplastidan lineages having plastids of secondary origin, although this discrepancy may be due to other factors, such as incomplete lineage sorting [42], limitations of phylogenetic inference methods [43], or any combination of the above items.

\section{No distinct nucleocytoplasmic traits uniting the three "primary"-plastid bearing lineages are known}

In addition to a lack of resolution for archaeplastidan phylogeny based on analyses of nucleocytoplasmic gene markers, no distinct nucleocytoplasmic morphological traits that unite the three "primary"-plastid containing groups to the exclusion of other groups are known [44,45]. As far as we are aware, the Archaeplastida hypothesis is based on traits associated with the plastid component only and, more importantly, the assumption that the plastids of the three groups comprising Archaeplastida are of primary origin. In other words, if the plastids and their associated traits are stripped from the cells, we are left with few to no clues as to the affinity of the three archaeplastidan groups in comparison to other eukaryotic groups. This does not exclude those shared traits that originated via lateral gene transfer from non-cyanobactierial sources, such as the presumed chlamydial symbiont ([17,46], but see discussion in [47]). Most of such shared traits (or genes) are nevertheless related to plastid function, such as starch synthesis, and have been shown to be capable of being serially transferred via secondary/tertiary plastid-generating endosymbiosis [48]. While one or more genes (e.g. thiamine pyrophosphate-dependent pyruvate decarboxylase family protein) that may not be related to plastid evolution or function have been reported to support archaeplastidan monophyly [17], the number of such incidences is low enough that they could have occurred by chance alone, especially given that thousands of genes have been analyzed [49]. When massive amounts of data are analyzed and a conclusion is to be drawn in a quantitative manner, it is imperative to perform control analyses to estimate the level of background noise, which unfortunately does not seem to have been implemented in many studies.

It should be noted that the presence of two membranes surrounding the plastid is the main argument for a primary origin of the plastids in Chloroplastida as well as those in Rhodophyta and Glaucophyta; nonetheless, it is conceivable that secondary or tertiary plastids could be bound by only two envelope membranes. Some dinoflagellates, for example, have two membranes surrounding their plastids even though their organelles are clearly not of primary origin [2]. Additionally, kleptoplastidy, such as that known from the sea slug Elysia chlorotica, which steals and keeps plastids of the stramenopile Vaucheria litorea and is known to strip off the outer (third) membrane during plastid acquisition [50], provides another possible mechanism for membrane loss from secondary or tertiary plastids. 


\section{Plastids of green algae and plants have characteristics that might be suggestive of their possible secondary origins}

Plastid genomes are greatly reduced in size and gene content compared to those of free-living cyanobacteria [1]. It is estimated that more than $90 \%$ of plastid genes were lost or transferred to the host nucleus as the cyanobacterial endosymbiont was incorporated into the host and eventually transformed into an organelle [51]. Plastid genomes of photosynthetic algae code for up to around 200 proteins as well as a handful of tRNAs and rRNAs (Fig. 1). Of plastid-bearing lineages, the most gene-rich plastid genomes are found in the red algae; phototrophic red algae encode 164-199 proteins in their plastid genomes. Secondary plastids of red algal origin, including those harbored by photosynthetic members of the cryptophytes, haptophytes, and stramenopiles (note that some of these lineages may have acquired their plastids "serially" from algae with red algal-derived plastids [8,52]), carry 100-150 protein-coding genes, less than what the plastids of autotrophic red algae encode (Fig. 1). The dinoflagellate Karlodinium veneficum, which harbors a tertiary plastid of haptophyte origin, encodes only 56 proteins in its plastid genome; by comparison, at least 100 proteins are encoded in plastid genomes of haptophytes (Fig. 1). As such, the pattern seems to be that the serial transfer of a plastid into a new host eukaryote is often accompanied by a measurable loss of genes in the plastid genome. Of the three groups comprising Archaeplastida, members of the Chloroplastida encode the least number of genes in their plastids: no more than 100 proteins are encoded in green algal and plant plastid genomes (Fig. 1). These observations suggest that, of the three "primary"-plastid bearing groups, Chloroplastida is the most likely candidate for an algal lineage originating from a secondary plastid-generating event.

In addition, the lack of phycobilisomes (i.e. light harvesting protein complexes that are anchored to, and physically separate, the thylakoid membranes of some algae) in Chloroplastida [2] favors the hypothesis that their plastids are more likely to be of secondary origin than are the plastids of red algae or glaucophytes. This is supported by the fact that none of the plastids that have evolved secondarily (or possibly tertiarily) from red algae possess structurally intact phycobilisomes [2]. In fact, red algae and glaucophytes are the only eukaryotic algae with phycobilisomes [2]. Cryptophytes, which acquired plastids from a red algal symbiont, produce pigment components of phycobilisomes, such as phycoerythrin or phycocyanin; however, they nevertheless are incapable of assembling intact phycobilisomes, and hence display stacked thylakoid membranes [2].

Additionally, plastidal storage of starch in green algae and land plants, as opposed to the assumed ancestral cytosolic location of starch, seems to be indicative of the occurrence of an evolutionary event by a quantum leap - comparable to the acquisition of a plastid - at the point of origin of the Chloroplastida. By comparison, red algae and glaucophytes possess a cytosolic starch metabolism, which is simpler than the starch metabolism of green algae and land plants [53]. Deschamps et al. [54] and Cenci et al. [53] described at length the difficulties with explaining the return of starch metabolism from the cytoplasm into the plastid as green algae diverged from red or glaucophyte-like algae. In our opinion, an independent, secondary endosymbiotic plastidgenerating event at the ancestry of the Chloroplastida better explains why this massive rewiring of carbon metabolism - which is difficult to explain by a more gradual mode of evolution - became necessary.

\section{Retention of phagocytosis in at least some members of green algae suggests the possibility that the green algae + land plant lineage may not be as old as the red algae or glaucophytes}

Phagocytosis refers to the process of engulfing relatively large particulate matter ( $>4 \mu \mathrm{m}$ in diameter), and is performed only by eukaryotic cells [55]. Phagocytosis is widely distributed across the tree of eukaryotes, and hence was likely present in the last eukaryotic common ancestor (LECA); however, it has been lost on multiple occasions, especially in those lineages where plastids (and therefore photosynthesis) were acquired, and in which nutritional uptake by phagotrophy thereby became dispensable [56]. Consequently, whereas phagocytosis was an essential step toward the endosymbiotic origin of plastids, neither extant red algae nor extant glaucophytes are capable of feeding on bacteria or other microbes for nutrition [56]. Phagocytosis appears to be better retained in photosynthetic eukaryotes harboring secondary or tertiary plastids. These include phago-mixotrophic members of the chlorarachniophytes, cryptophytes, dinoflagellates, euglenophytes, haptophytes, and stramenopiles $[57,58]$. Better retention of phagotrophy might be partially explained by a relatively shorter time that has elapsed since secondary- or tertiary-plastid bearing lineages became phototrophic; in other words, with less time, there is more chance that phagocytosis has been retained.

In contrast to red algae and glaucophytes, at least a few members of the green algae, including the marine prasinophytes Cymbomonas and Micromonas, have been shown to have the capacity to feed on bacteria $[59,60]$. While the selective pressure keeping these algae phago-mixotrophic is not well understood, it is tempting to speculate that green algal phagocytosis may be indicative of a relatively young age of the Chloroplastida lineage compared to that of red or glaucophyte algae.

\section{Concluding remarks}

Our views of eukaryotic evolutionary relationships are constantly evolving. The consolidation of all purportedly primary-plastid-bearing lineages into Archaeplastida is one of the more readily appreciated and widely accepted such views. Yet, as we have seen, this picture is not as certain as is often represented. Large-scale phylogenetic approaches thus far have failed to reach a consensus on the Archaeplastida hypothesis. Such lack of phylogenetic signal for this otherwise widely accepted hypothesis may be explained by one or two of the three archaeplastidan lineages having plastids of secondary origins [13]. Based on some cellular features, 


\section{Total number of protein-coding genes in plastid genomes}

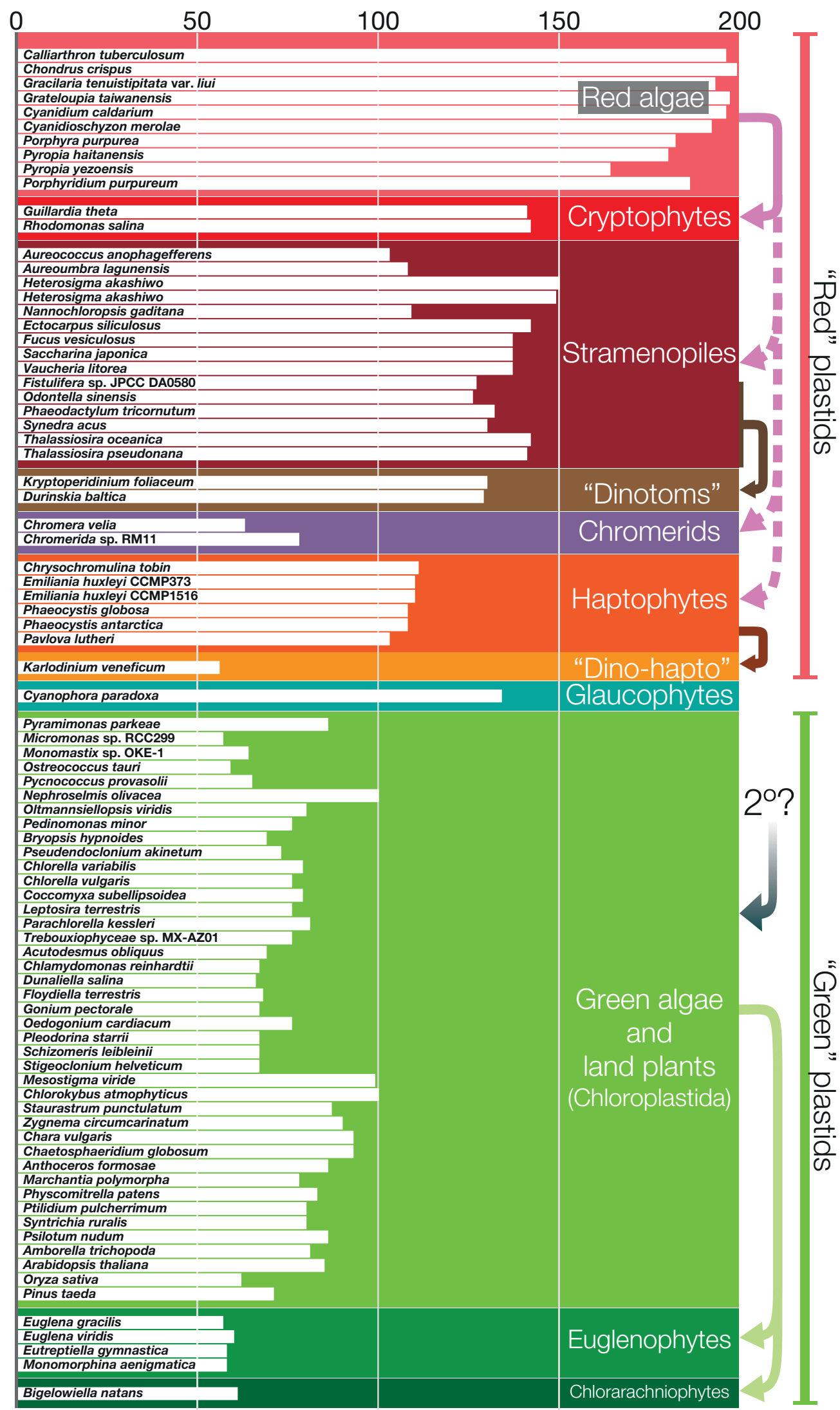

Fig. 1 The total number of predicted protein-coding genes in completely sequenced plastid genomes of photosynthetic eukaryotes. Duplicated genes were counted only once, and only conserved genes were tallied; those predicted proteins of unknown function that are unique to a terminal taxon were excluded from the analysis. Our calculations are based on plastid genome annotation information available through the Chloroplast Genome Database (CpBase; http://chloroplast.ocean.washington.edu) or the National Center for Biotechnology Information (NCBI; http://www.ncbi.nlm.nih.gov). Distinct algal groups are color-coded. "Dinotoms" and "Dino-hapto" refer to dinoflagellates that harbor plastids of diatom and haptophyte origins, respectively. Arrows indicate the direction of plastid transfers via secondary or tertiary (or possibly quaternary or greater) endosymbioses. 
including the way the carbohydrate biochemistry of the organelle is incorporated into that of the host eukaryotes, we suggest that the Chloroplastida are the best candidate for an archeaplastidan group arising from a secondary plastidgenerating event. Future investigations that would improve our understanding on this topic include $(i)$ a phylogenomic analysis similar to the falsification method devised by Baurain et al. [61] to test competing hypotheses concerning archaeplastidan phylogeny, (ii) fine detailed characterization

\section{Acknowledgments}

A Heiss and J Burns are thanked for their critical comments on this manuscript. This work was supported by the AMNH's startup funds awarded to EK; and the JSPS's grant-in-aid for scientific research funds (\#25891027 and \#26117731) and funds from the Institute for Fermentation, Osaka, awarded to SM.

\section{Authors' contributions}

The following declarations about authors' contributions to the research have been made: conceived the project and wrote the manuscript together: EK, SM.

\section{Competing interests}

No competing interests have been declared.

\section{References}

1. Archibald JM. The puzzle of plastid evolution. Curr Biol. 2009;19(2):R81-R88. http://dx.doi.org/10.1016/j.cub.2008.11.067

2. Graham LE. Algae. 2nd ed. San Francisco, CA: Pearson/Benjamin Cummings; 2009.

3. Palmer JD. The symbiotic birth and spread of plastids: how many times and whodunit? J Phycol. 2003;39(1):4-12. http://dx.doi. org/10.1046/j.1529-8817.2003.02185.x

4. Howe C, Barbrook A, Nisbet RE, Lockhart P, Larkum AW. The origin of plastids. Philos Trans R Soc Lond B Biol Sci. 2008;363(1504):26752685. http://dx.doi.org/10.1098/rstb.2008.0050

5. Cavalier-Smith T. Kingdom protozoa and its 18 phyla. Microbiol Rev. 1993;57(4):953-994.

6. Adl SM, Simpson AGB, Lane CE, Lukeš J, Bass D, Bowser SS, et al. The revised classification of eukaryotes. J Eukaryot Microbiol. 2012;59(5):429-514. http://dx.doi. org/10.1111/j.1550-7408.2012.00644.x

7. Minge MA, Shalchian-Tabrizi K, Tørresen OK, Takishita K, Probert I, Inagaki Y, et al. A phylogenetic mosaic plastid proteome and unusual plastid-targeting signals in the green-colored dinoflagellate Lepidodinium chlorophorum. BMC Evol Biol. 2010;10(1):191. http:// dx.doi.org/10.1186/1471-2148-10-191

8. Sanchez-Puerta MV, Bachvaroff TR, Delwiche CF. Sorting wheat from chaff in multi-gene analyses of chlorophyll $c$-containing plastids. Mol Phylogenet Evol. 2007;44(2):885-897. http://dx.doi.org/10.1016/j. ympev.2007.03.003

9. Petersen J, Ludewig AK, Michael V, Bunk B, Jarek M, Baurain D, et al. Chromera velia, endosymbioses and the rhodoplex hypothesis - plastid evolution in cryptophytes, alveolates, stramenopiles, and haptophytes (CASH lineages). Genome Biol Evol. 2014;6(3):666-684. http://dx.doi. org/10.1093/gbe/evu043

10. Parfrey LW, Lahr DJG, Knoll AH, Katz LA. Estimating the timing of early eukaryotic diversification with multigene molecular clocks. Proc Natl Acad Sci USA. 2011;108(33):13624-13629. http://dx.doi. org/10.1073/pnas.1110633108

11. Burki F, Okamoto N, Pombert JF, Keeling PJ. The evolutionary history of haptophytes and cryptophytes: phylogenomic evidence for separate origins. Proc Biol Sci. 2012;279(1736):2246-2254. http:// dx.doi.org/10.1098/rspb.2011.2301

12. Stiller JW, Hall BD. The origin of red algae: implications for plastid evolution. Proc Natl Acad Sci USA. 1997;94(9):4520-4525. of glaucophyte cytology (e.g. flagellar apparatus) - which is currently limited-for comparative analysis of cell morphology among "primary" plastid containing groups compared to other eukaryotic groups, and (iii) identification and characterization of deeply branching novel eukaryotic taxa, especially those that are related to the archaeplastidan groups, and hence could improve the resolution of archaeplastidan phylogeny.

13. Stiller JW. Toward an empirical framework for interpreting plastid evolution. J Phycol. 2014;50(3):462-471. http://dx.doi.org/10.1111/ jpy. 12178

14. Douglas SE, Turner S. Molecular evidence for the origin of plastids from a cyanobacterium-like ancestor. J Mol Evol. 1991;33(3):267-273. http://dx.doi.org/10.1007/BF02100678

15. Morden CW, Delwiche CF, Kuhsel M, Palmer JD. Gene phylogenies and the endosymbiotic origin of plastids. Biosystems. 1992;28(1-3):7590. http://dx.doi.org/10.1016/0303-2647(92)90010-V

16. Criscuolo A, Gribaldo S. Large-scale phylogenomic analyses indicate a deep origin of primary plastids within cyanobacteria. Mol Biol Evol. 2011;28(11):3019-3032. http://dx.doi.org/10.1093/molbev/msr108

17. Price DC, Chan CX, Yoon HS, Yang EC, Qiu H, Weber APM, et al. Cyanophora paradoxa genome elucidates origin of photosynthesis in algae and plants. Science. 2012;335(6070):843-847. http://dx.doi. org/10.1126/science.1213561

18. Baldauf SL. A kingdom-level phylogeny of eukaryotes based on combined protein data. Science. 2000;290(5493):972-977. http:// dx.doi.org/10.1126/science.290.5493.972

19. Moreira D, Le Guyader H, Philippe H. The origin of red algae and the evolution of chloroplasts. Nature. 2000;405(6782):69-72. http:// dx.doi.org/10.1038/35011054

20. Rodríguez-Ezpeleta N, Brinkmann H, Burey SC, Roure B, Burger G, Löffelhardt W, et al. Monophyly of primary photosynthetic eukaryotes: green plants, red algae, and glaucophytes. Curr Biol. 2005;15(14):13251330. http://dx.doi.org/10.1016/j.cub.2005.06.040

21. Rodríguez-Ezpeleta N, Brinkmann H, Burger G, Roger AJ, Gray MW, Philippe $\mathrm{H}$, et al. Toward resolving the eukaryotic tree: the phylogenetic positions of jakobids and cercozoans. Curr Biol. 2007;17(16):14201425. http://dx.doi.org/10.1016/j.cub.2007.07.036

22. Burki F, Shalchian-Tabrizi K, Minge M, Skjæveland Å, Nikolaev SI, Jakobsen KS, et al. Phylogenomics reshuffles the eukaryotic supergroups. PLoS ONE. 2007;2(8):e790. http://dx.doi.org/10.1371/ journal.pone.0000790

23. Nozaki H, Iseki M, Hasegawa M, Misawa K, Nakada T, Sasaki N, et al. Phylogeny of primary photosynthetic eukaryotes as deduced from slowly evolving nuclear genes. Mol Biol Evol. 2007;24(8):1592-1595. http://dx.doi.org/10.1093/molbev/msm091

24. Burki F, Shalchian-Tabrizi K, Pawlowski J. Phylogenomics reveals a new "megagroup" including most photosynthetic eukaryotes. Biol Lett. 2008;4(4):366-369. http://dx.doi.org/10.1098/rsbl.2008.0224

25. Burki F, Inagaki Y, Brate J, Archibald JM, Keeling PJ, Cavalier-Smith $\mathrm{T}$, et al. Large-scale phylogenomic analyses reveal that two enigmatic protist lineages, telonemia and centroheliozoa, are related to photosynthetic chromalveolates. Genome Biol Evol. 2010;1:231-238. http:// dx.doi.org/10.1093/gbe/evp022

26. Nozaki H, Maruyama S, Matsuzaki M, Nakada T, Kato S, Misawa K. Phylogenetic positions of Glaucophyta, green plants (Archaeplastida) and Haptophyta (Chromalveolata) as deduced from slowly evolving nuclear genes. Mol Phylogenet Evol. 2009;53(3):872-880. http://dx.doi. org/10.1016/j.ympev.2009.08.015

27. Brown MW, Kolisko M, Silberman JD, Roger AJ. Aggregative multicellularity evolved independently in the eukaryotic supergroup rhizaria. Curr Biol. 2012;22(12):1123-1127. http://dx.doi.org/10.1016/j. cub.2012.04.021

28. Zhao S, Burki F, Brate J, Keeling PJ, Klaveness D, Shalchian-Tabrizi K. 
Collodictyon - an ancient lineage in the tree of eukaryotes. Mol Biol Evol. 2012;29(6):1557-1568. http://dx.doi.org/10.1093/molbev/mss001

29. Brown MW, Sharpe SC, Silberman JD, Heiss AA, Lang BF, Simpson AGB, et al. Phylogenomics demonstrates that breviate flagellates are related to opisthokonts and apusomonads. Proc Biol Sci. 2013;280(1769):20131755. http://dx.doi.org/10.1098/rspb.2013.1755

30. Burki F, Corradi N, Sierra R, Pawlowski J, Meyer GR, Abbott CL, et al. Phylogenomics of the intracellular parasite Mikrocytos mackini reveals evidence for a mitosome in rhizaria. Curr Biol. 2013;23(16):1541-1547. http://dx.doi.org/10.1016/j.cub.2013.06.033

31. Yabuki A, Kamikawa R, Ishikawa SA, Kolisko M, Kim E, Tanabe AS, et al. Palpitomonas bilix represents a basal cryptist lineage: insight into the character evolution in Cryptista. Sci Rep. 2014;4:4641. http:// dx.doi.org/10.1038/srep04641

32. Jackson CJ, Reyes-Prieto A. The mitochondrial genomes of the glaucophytes Gloeochaete wittrockiana and Cyanoptyche gloeocystis: multilocus phylogenetics suggests a monophyletic Archaeplastida. Genome Biol Evol. 2014;6(10):2774-2785. http://dx.doi.org/10.1093/ gbe/evu218

33. Stiller JW, Riley J, Hall BD. Are red algae plants? A critical evaluation of three key molecular data sets. J Mol Evol. 2001;52(6):527-539. http://dx.doi.org/10.1007/s002390010183

34. Oudot-Le Secq MP, Grimwood J, Shapiro H, Armbrust EV, Bowler C, Green BR. Chloroplast genomes of the diatoms Phaeodactylum tricornutum and Thalassiosira pseudonana: comparison with other plastid genomes of the red lineage. Mol Genet Genomics. 2007;277(4):427-439. http://dx.doi.org/10.1007/s00438-006-0199-4

35. Kim E, Lane CE, Curtis BA, Kozera C, Bowman S, Archibald JM. Complete sequence and analysis of the mitochondrial genome of Hemiselmis andersenii CCMP644 (Cryptophyceae). BMC Genomics. 2008;9(1):215. http://dx.doi.org/10.1186/1471-2164-9-215

36. Chan CX, Yang EC, Banerjee T, Yoon HS, Martone PT, Estevez JM, et al. Red and green algal monophyly and extensive gene sharing found in a rich repertoire of red algal genes. Curr Biol. 2011;21(4):328-333. http://dx.doi.org/10.1016/j.cub.2011.01.037

37. Adl SM, Simpson AGB, Farmer MA, Andersen RA, Anderson OR, Barta JR, et al. The new higher level classification of eukaryotes with emphasis on the taxonomy of protists. J Eukaryot Microbiol. 2005;52(5):399-451. http://dx.doi.org/10.1111/j.1550-7408.2005.00053.x

38. Cavalier-Smith T. Megaphylogeny, cell body plans, adaptive zones: causes and timing of eukaryote basal radiations. J Eukaryot Microbiol. 2009;56(1):26-33. http://dx.doi.org/10.1111/j.1550-7408.2008.00373.x

39. Roger AJ, Simpson AGB. Evolution: revisiting the root of the eukaryote tree. Curr Biol. 2009;19(4):R165-R167. http://dx.doi.org/10.1016/j. cub.2008.12.032

40. Walker G, Dorrell RG, Schlacht A, Dacks JB. Eukaryotic systematics: a user's guide for cell biologists and parasitologists. Parasitology. 2011;138(13):1638-1663. http://dx.doi.org/10.1017/ S0031182010001708

41. Deschamps P, Moreira D. Signal conflicts in the phylogeny of the primary photosynthetic eukaryotes. Mol Biol Evol. 2009;26(12):27452753. http://dx.doi.org/10.1093/molbev/msp189

42. Maddison W, Knowles L. Inferring phylogeny despite incomplete lineage sorting. Syst Biol. 2006;55(1):21-30. http://dx.doi. org/10.1080/10635150500354928

43. Laurin-Lemay S, Brinkmann H, Philippe H. Origin of land plants revisited in the light of sequence contamination and missing data. Curr Biol. 2012;22(15):R593-R594. http://dx.doi.org/10.1016/j. cub.2012.06.013

44. O'Kelly CJ. Relationships of eukaryotic algal groups to other protists. In: Berner T, editor. Ultrastructure of microalgae. Boca Raton, FL: CRC Press; 1993. p. 269-293.
45. Stiller JW. Plastid endosymbiosis, genome evolution and the origin of green plants. Trends Plant Sci. 2007;12(9):391-396. http://dx.doi. org/10.1016/j.tplants.2007.08.002

46. Ball SG, Subtil A, Bhattacharya D, Moustafa A, Weber APM, Gehre L, et al. Metabolic effectors secreted by bacterial pathogens: essential facilitators of plastid endosymbiosis? Plant Cell. 2013;25(1):7-21. http://dx.doi.org/10.1105/tpc.112.101329

47. Rujan T, Martin W. How many genes in Arabidopsis come from cyanobacteria? An estimate from 386 protein phylogenies. Trends Genet. 2001;17(3):113-120. http://dx.doi.org/10.1016/S0168-9525(00)02209-5

48. Deschamps P, Haferkamp I, Dauvillee D, Haebel S, Steup M, Buleon A, et al. Nature of the periplastidial pathway of starch synthesis in the cryptophyte Guillardia theta. Eukaryot. Cell. 2006;5(6):954-963. http://dx.doi.org/10.1128/EC.00380-05

49. Dagan T, Martin W. The tree of one percent. Genome Biol. 2006;7(10):118. http://dx.doi.org/10.1186/gb-2006-7-10-118

50. Rumpho ME. Solar-powered sea slugs. Mollusc/algal chloroplast symbiosis. Plant Physiol. 2000;123(1):29-38. http://dx.doi.org/10.1104/ pp.123.1.29

51. Martin W, Rujan T, Richly E, Hansen A, Cornelsen S, Lins T, et al. Evolutionary analysis of Arabidopsis, cyanobacterial, and chloroplast genomes reveals plastid phylogeny and thousands of cyanobacterial genes in the nucleus. Proc Natl Acad Sci USA. 2002;99(19):1224612251. http://dx.doi.org/10.1073/pnas.182432999

52. Bodył A. Do plastid-related characters support the chromalveolate hypothesis? J Phycol. 2005;41(3):712-719. http://dx.doi. org/10.1111/j.1529-8817.2005.00091.x

53. Cenci U, Nitschke F, Steup M, Minassian BA, Colleoni C, Ball SG. Transition from glycogen to starch metabolism in Archaeplastida. Trends Plant Sci. 2014;19(1):18-28. http://dx.doi.org/10.1016/j. tplants.2013.08.004

54. Deschamps P, Haferkamp I, d' Hulst C, Neuhaus HE, Ball SG. The relocation of starch metabolism to chloroplasts: when, why and how. Trends Plant Sci. 2008;13(11):574-582. http://dx.doi.org/10.1016/j. tplants.2008.08.009

55. Flannagan RS, Jaumouillé V, Grinstein S. The cell biology of phagocytosis. Annu Rev Pathol. 2012;7(1):61-98. http://dx.doi.org/10.1146/ annurev-pathol-011811-132445

56. Raven JA, Beardall J, Flynn KJ, Maberly SC. Phagotrophy in the origins of photosynthesis in eukaryotes and as a complementary mode of nutrition in phototrophs: relation to Darwin's insectivorous plants. J Exp Bot. 2009;60(14):3975-3987. http://dx.doi.org/10.1093/jxb/erp282

57. Raven JA. Phagotrophy in phototrophs. Limnol Ocean. 1997;42(1):198205. http://dx.doi.org/10.4319/lo.1997.42.1.0198

58. Yamaguchi A, Yubuki N, Leander BS. Morphostasis in a novel eukaryote illuminates the evolutionary transition from phagotrophy to phototrophy: description of Rapaza viridis $\mathrm{n}$. gen. et $\mathrm{sp}$. (Euglenozoa, Euglenida). BMC Evol Biol. 2012;12(1):29. http://dx.doi. org/10.1186/1471-2148-12-29

59. Maruyama S, Kim E. A modern descendant of early green algal phagotrophs. Curr Biol. 2013;23(12):1081-1084. http://dx.doi.org/10.1016/j. cub.2013.04.063

60. McKie-Krisberg ZM, Sanders RW. Phagotrophy by the picoeukaryotic green alga Micromonas: implications for Arctic Oceans. ISME J. 2014;8(10):1953-1961. http://dx.doi.org/10.1038/ismej.2014.16

61. Baurain D, Brinkmann H, Petersen J, Rodriguez-Ezpeleta N, Stechmann A, Demoulin V, et al. Phylogenomic evidence for separate acquisition of plastids in cryptophytes, haptophytes, and stramenopiles. Mol Biol Evol. 2010;27(7):1698-1709. http://dx.doi.org/10.1093/ molbev/msq059 